\title{
Automatic detection of aorto-femoral vessel trajectory from whole-body computed tomography angiography data sets
}

\author{
Xinpei Gao ${ }^{1} \cdot$ Pieter H. Kitslaar ${ }^{1,6} \cdot$ Ricardo P. J. Budde $^{2} \cdot$ Shengxian Tu $^{3}$. \\ Michiel A. de Graaf ${ }^{5} \cdot \operatorname{Liang} \mathrm{Xu}^{4} \cdot \mathrm{Bo} \mathrm{Xu}^{4} \cdot$ Arthur J. H. A. Scholte ${ }^{5}$. \\ Jouke Dijkstra ${ }^{1} \cdot$ Johan H. C. Reiber ${ }^{1,6}$
}

Received: 10 December 2015 / Accepted: 22 April 2016 / Published online: 21 May 2016

(C) The Author(s) 2016. This article is published with open access at Springerlink.com

\begin{abstract}
Extraction of the aorto-femoral vessel trajectory is important to utilize computed tomography angiography (CTA) in an integrated workflow of the image-guided work-up prior to trans-catheter aortic valve replacement (TAVR). The aim of this study was to develop a new, fullyautomated technique for the extraction of the entire arterial access route from the femoral artery to the aortic root. An automatic vessel tracking algorithm was first used to find the centerline that connected the femoral accessing points and the aortic root. Subsequently, a deformable 3D-model fitting method was used to delineate the lumen boundary of the vascular trajectory in the whole-body CTA dataset. A validation was carried out by comparing the automatically obtained results with semi-automatically obtained results from two experienced observers. The whole framework was validated on whole body CTA datasets of 36 patients.
\end{abstract}

Pieter H. Kitslaar

P.H.Kitslaar@lumc.nl

1 Division of Image Processing, Department of Radiology, Leiden University Medical Center, 9600, 2300 RC Leiden, The Netherlands

2 Department of Radiology, University Medical Center Rotterdam, Rotterdam, The Netherlands

3 Biomedical Instrument Institute, School of Biomedical Engineering, Shanghai Jiao Tong University, Shanghai, China

4 Catheterization Laboratory, National Center for Cardiovascular Diseases of China and Fu Wai Hospital, Beijing, China

5 Department of Cardiology, Leiden University Medical Center, Leiden, The Netherlands

6 Medis medical imaging systems b.v., Leiden, The Netherlands
The average Dice similarity indexes between the segmentations of the automatic method and observer 1 for the left ilio-femoral artery, the right ilio-femoral artery and the aorta were $0.977 \pm 0.030,0.980 \pm 0.019,0.982 \pm 0.016$; the average Dice similarity indexes between the segmentations of the automatic method and observer 2 were $0.950 \pm 0.040$, $0.954 \pm 0.031$ and $0.965 \pm 0.019$, respectively. The interobserver variability resulted in a Dice similarity index of $0.954 \pm 0.038,0.952 \pm 0.031$ and $0.969 \pm 0.018$ for the left iliofemoral artery, the right ilio-femoral artery and the aorta. The average minimal luminal diameters (MLDs) of the ilio-femoral artery were $6.03 \pm 1.48,5.70 \pm 1.43$ and $5.52 \pm 1.32 \mathrm{~mm}$ for the automatic method, observer 1 and observer 2 respectively. The MLDs of the aorta were $13.43 \pm 2.54,12.40 \pm 2.93$ and $12.08 \pm 2.40 \mathrm{~mm}$ for the automatic method, observer 1 and observer 2 respectively. The automatic measurement overestimated the MLD slightly in the ilio-femoral artery at the average by $0.323 \mathrm{~mm}(\mathrm{SD}=0.49 \mathrm{~mm}, \mathrm{p}<0.001)$ compared to observer 1 and by $0.51 \mathrm{~mm}(\mathrm{SD}=0.71 \mathrm{~mm}, \mathrm{p}<0.001)$ compared to observer 2 . The proposed segmentation approach can automatically provide reliable measurements of the entire arterial accessing route that can be used to support TAVR procedures. To the best of our knowledges, this approach is the first fully automatic segmentation method of the whole aorto-femoral vessel trajectory in CTA images.

Keywords CTA - TAVR · Aorto-femoral ·

Segmentation $\cdot$ Fully-automatic

\section{Introduction}

Aortic valve stenosis is a disease particularly prevalent among senior citizens over the age of 65 years [1]. If left untreated, it is associated with a significant mortality. 
Historically surgical aortic valve replacement (SAVR) was used to treat this disease. Unfortunately, not all patients are suitable for such a procedure. Especially, SAVR may be associated with a high perioperative mortality risk in elderly patients $[1,2]$. Trans-catheter aortic valve replacement (TAVR)/trans-catheter aortic valve implantation (TAVI) has been developed as a therapeutic option during the last decade for the inoperable or very high-risk patients [3].

To minimize the procedure-related complications, special attention should be given to the selection procedure to decide which patients are suitable TAVR candidates. Computed tomography (CT) imaging has been proven to be able to predict vascular complications among patients undergoing trans-femoral trans-catheter aortic valve replacement (TF-TAVR) [4]. Evaluation of the size and tortuosity of the ilio-femoral arteries is required to determine the feasibility of a transfemoral (TF) approach [5, 6]. Furthermore, the size of the entire aorta is also needed for proper device selection in TAVR $[7,8]$. To integrate such measurements seamlessly and conveniently into the TAVR clinical workflow, a fully automatic framework was developed, allowing detection of the femoral/aorta access route and the vessel sizes based on 3D contour detection approaches.

In the literature, several approaches have been described for the detection of the arterial contours in computed tomography angiography (CTA) data sets. Lesage et al. [9] reviewed the state-of-the-art on vascular segmentation in multi-modality images. The model-based vascular segmentation approach is commonly used. In our study, the vessel surface was modeled by a centerline curve with a generalized cylinder. This cross-section model with prior shape information can reduce the complexity of the contour detection procedure [10].

The approach that we have taken consists of two steps (Fig. 1), firstly, a centerline extraction from the femoral arteries to the aortic root [11]; and secondly, a 3D contour detection approach using a subdivision surface model fitting method to accurately delineate the vascular access route [10]. Validation of this study was realized on 36 CTA datasets which were acquired during routine clinical practice prior to the TAVR procedures.

\section{Methods}

\section{Centerline extraction}

Since the details of the fully automatic centerline extraction algorithm were published in [11], we briefly summarize the steps below.

The CTA datasets were resampled at first to reduce the computation time. The centerline extraction step was executed on the resampled images. A wave-propagation algorithm was used, starting from the aortic arch which is detected automatically. To detect the aorta arch, prior knowledge about aorta diameter from clinical research [12] was integrated into a Gaussian probabilistic distribution model [13]. After calculating the probabilistic model, a probability map was generated. From a point in the aorta arch, the algorithm propagated in two directions. One side propagated into the aortic root, and the other side propagated into the femoral arteries. After the wave propagation, the result image was used as a weight image for the Dijkstra shortest path algorithm. The Dijkstra algorithm was executed twice. First, to extract the centerline from the left femoral artery end to the aortic root. Second, to extract the centerline from the right femoral artery end to the aortic root. After these two centerlines were found, the bifurcation point of the two centerlines was detected. Finally, the centerlines were split into three parts: the centerline in the aorta, in the left ilio-femoral artery and in the right ilio-femoral artery.

Next, the centerlines in the femoral arteries were improved by a centerline refinement step. For this, a multi-scale medialness response based wave-propagation scheme [14] was used. First, multi planar reconstruction (MPR) transversal image slice stacks were generated according to the initial centerline along the femoral artery. Next, the medialness response was computed from a circle centered at the initial
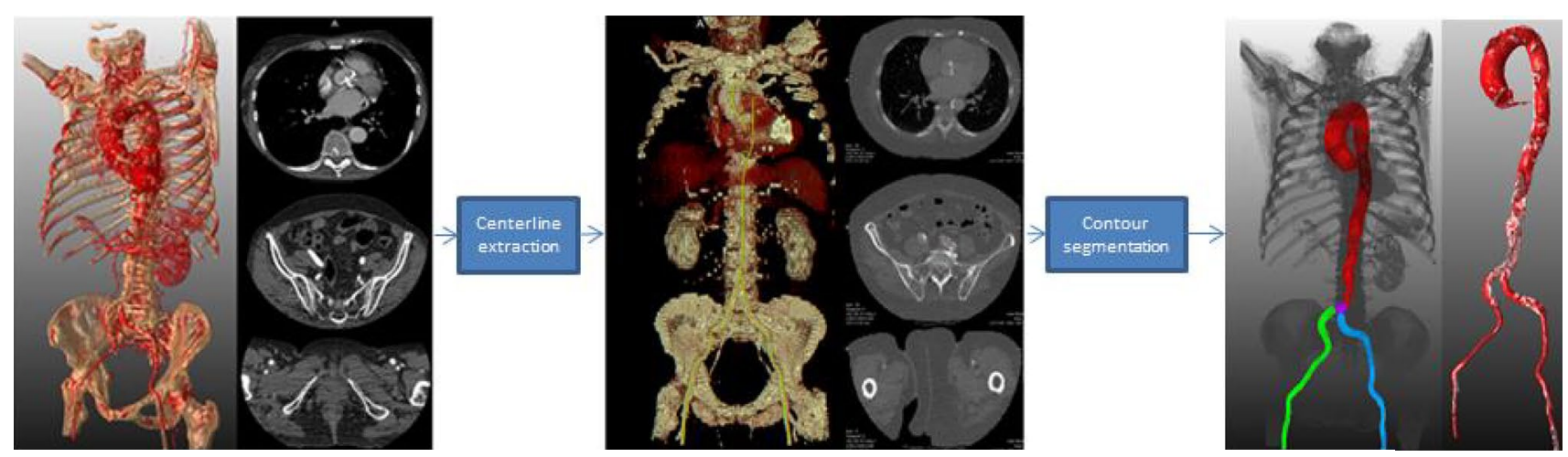

Fig. 1 The general pipeline of trajectory contour detection, including two steps: centerline extraction and contour detection 
centerline point, with multi-scale radius. This response was constructed as to give high values inside the center of the lumen and lower values inside calcifications. The response was used as the speed function for the wave propagation and back tracking method to generate the final refined centerline. Figure 2 shows an example of the comparison of the automatic extracted centerline and the refined centerline.

\section{Contour detection}

The vascular trajectory was segmented by a deformable model fitting method [10]. The deformable model was constructed from a coarse surface mesh representation created from the obtained centerlines. From this coarse mesh a smooth higher resolution surface mesh was created using a standard subdivision refinement scheme. This higher resolution subdivision surface was next deformed by iteratively updating the coarse mesh description by fitting to the lumen edge information in the CT scans. In [10], this model was applied in CTA images of the carotid artery including bifurcations, and provided good results. A big advantage of the subdivision surface approach is that it is able to model the bifurcation of the iliac arteries.

\section{Pre-processing}

In our study, whole-body CTA datasets were used. In these images different kinds of anatomic tissues can be distinguished. The surrounding background of the contrast-filled vessels is quite complex and may confuse the subdivision surface fitting. For our purposes, we distinguished two background categories: high intensity tissue (calcifications, bones, and metal artifacts), and low intensity tissue (muscles, fat, liver etc.). We masked out the surrounding background by an adaptive threshold method similar to Shahzad et al. [15].

This method is based on the assumption that the HU value (intensity) along the artery should be a smooth gradual decreasing curve when this artery is without the presence of calcium [16].

Based on this, an intensity profile of the voxels along our initially extracted centerline was extracted (Fig. 3). Next, a second order polynomial curve was fitted through the intensity profile data; this curve simulated the ideal case without calcium. Curve "b" in Fig. 3 shows this polynomial fit curve. Also shown is curve "a" which is an upward shifted version of curve "b" obtained by adding a constant value proportional to the standard deviation (SD) of the intensities in the profile. The values on curve "a" were used as the intensity threshold values for the high intensity structures (e.g. calcium) on the corresponding image slices along the vessel centerline. A similar method was used to find the tissue with lower intensity than the contrast-filled lumen using curve "c" which is a downward shifted version of curve " $b$ ". Figure 4 shows examples of the original and mask slices in the CTA data using this method.

\section{Model fitting}

The deformable model wasinitialized from the centerline curves as a generalized cylinder with local diameter
Fig. 2 Result of centerline refinement step. The red crosses are the original centerline points; the blue crosses are the refined centerline points. The blue points avoid the calcium, and are better located in the middle of the cross-section of the artery lumen compared to the red points

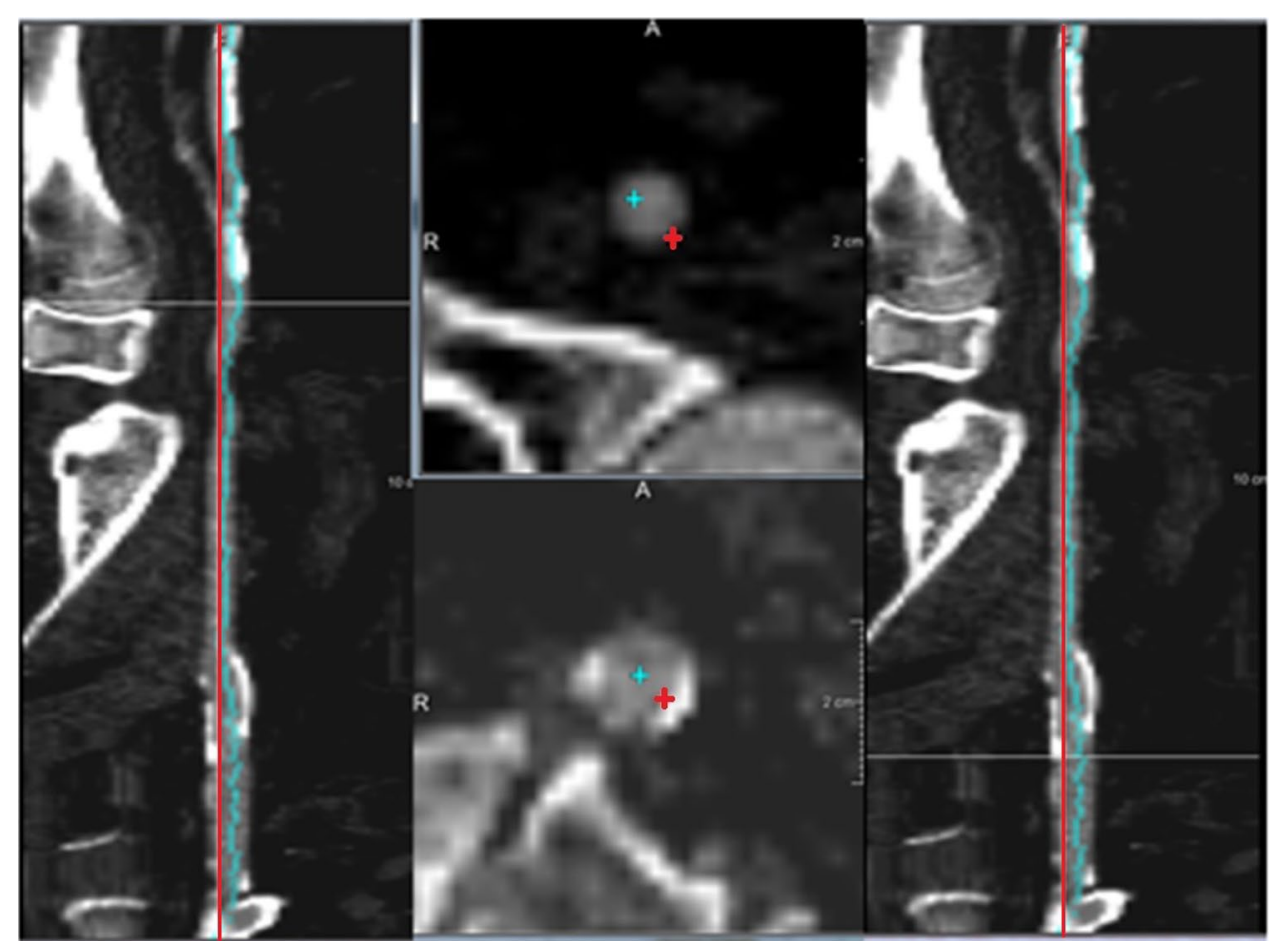


Fig. 3 Intensity profile along the centerline (yellow curve), polynomial curves (green). Yellow curve intensity values in $\mathrm{HU}$ of the voxels along the curves second order polynomial fit $(b)$ of the intensity values with standard deviation margin above $(a)$ and below $(c)$ centerline (in $\mathrm{mm}$ ). Green

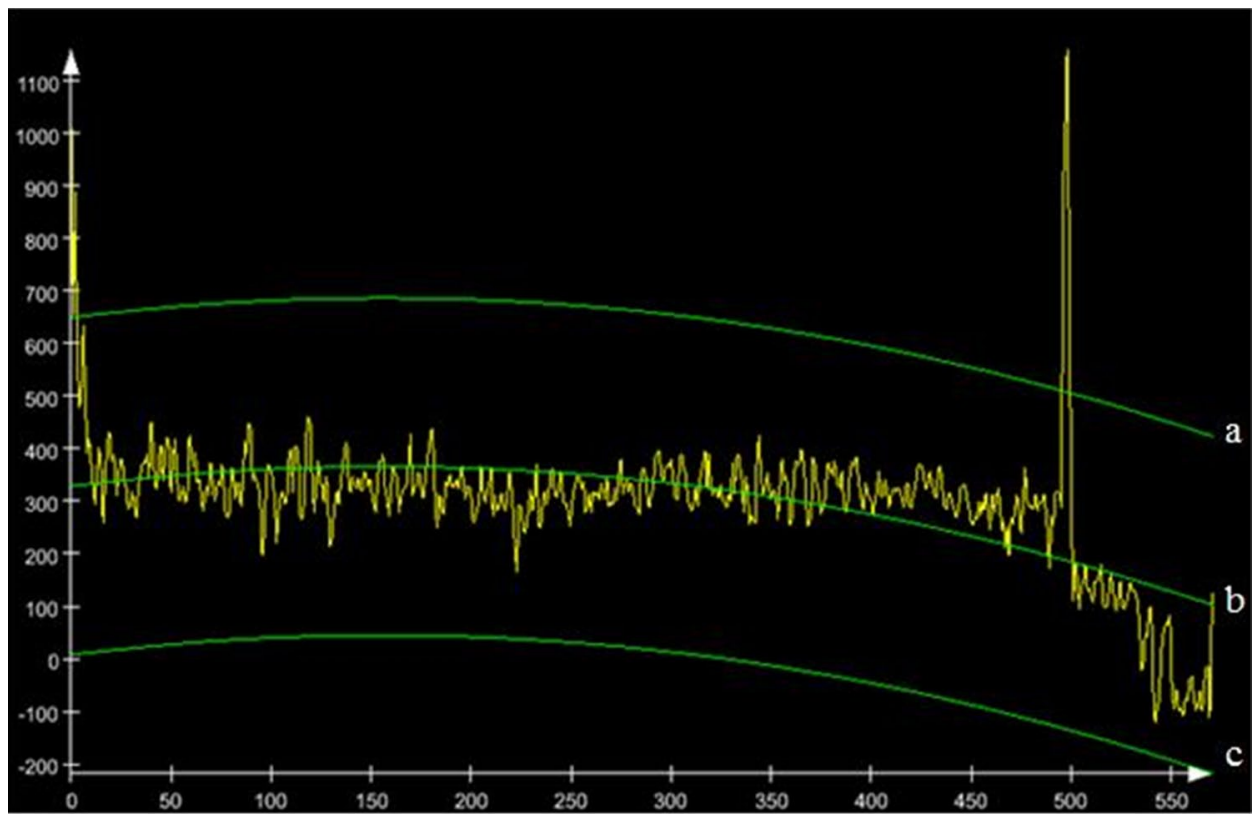

To compare the different segmentation results, the obtained 3D surface models were resliced along the centerlines to obtain planar 2D contours. From these contours, the cross sectional area and the minimal diameter were calculated as clinical parameters and compared between the different segmentation results. Also, the Dice similarity index (or coefficient) was calculated between the contours from the different segmentation results.

To investigate the influence of image quality, also the mean and standard deviation of the HU values in the descending aorta were measured for each subject.

\section{Statistical analysis}

The results of the clinical parameters from the automatic segmentations were compared with the results from the semi-automatic segmentations of the observers by the paired $t$ test. The Bland-Altman plots were calculated to quantify the mean error and the SD. The correlations were estimated by Pearson's correlation coefficient.

The statistical analyses were conducted with SPSS (version 20.0, SPSS Inc, Chicago, IL, USA) and MedCalc (version 15.6, Ostend, Belgium).

\section{Results}

\section{Segmentation evaluation}

To evaluate the automatic segmentation method, two experienced observers independently corrected the automatic segmentation result. This was done using a dedicated editing tool in which the automatically obtained surface model could interactively be modified.

\section{Data acquisition protocols}

This is a retrospective study; the datasets were acquired before this research project started as part of routine clinical care protocols. Only anonymous routine clinical datasets were used. 

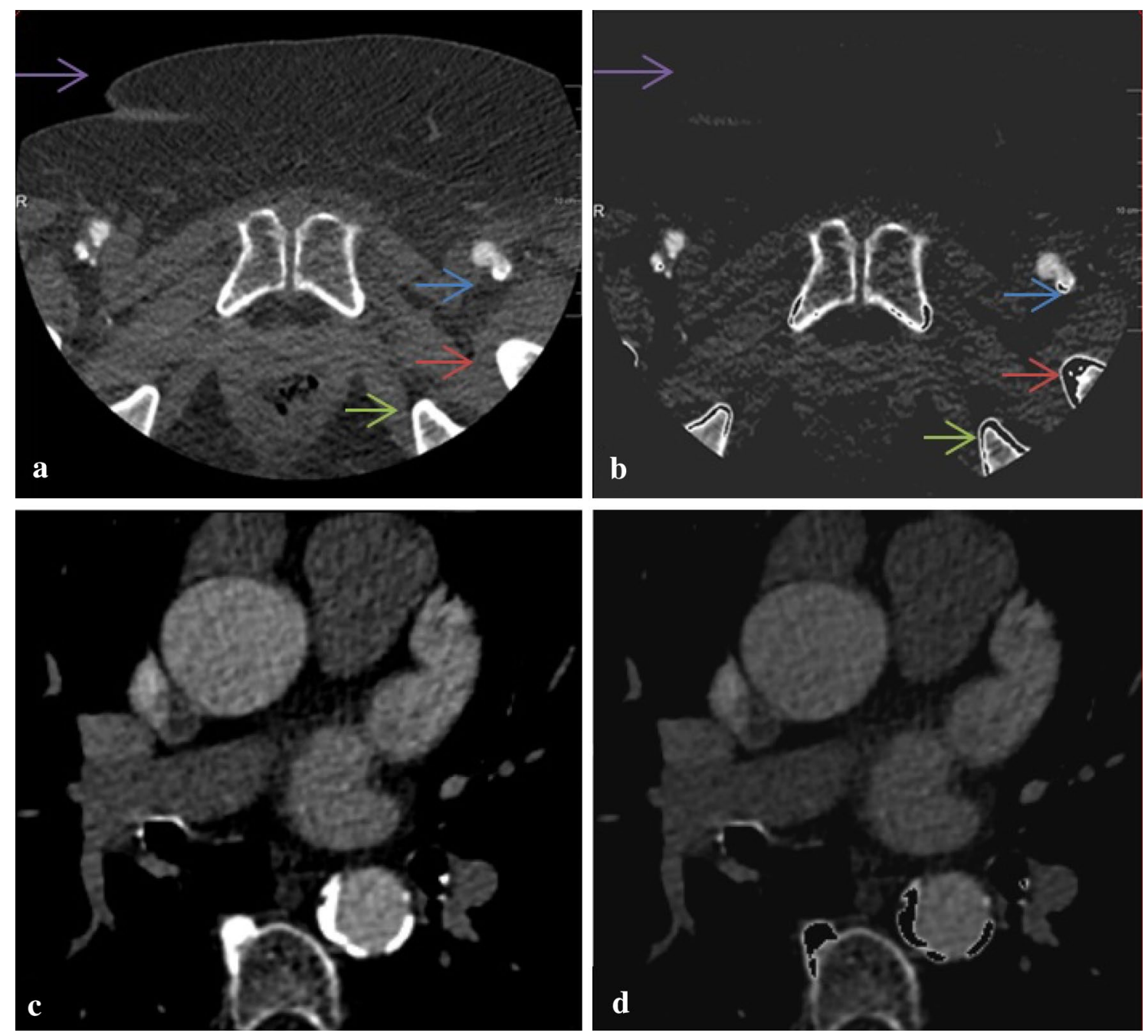

Fig. 4 Examples of pre-processed slices. a Original femoral artery image. b Processed femoral artery image. c Original aorta image. low intensity tissue that has been removed in processed image, other arrows indicate the processed high intensity tissue d Processed aorta image. The purple arrows in (a) and (b) indicate
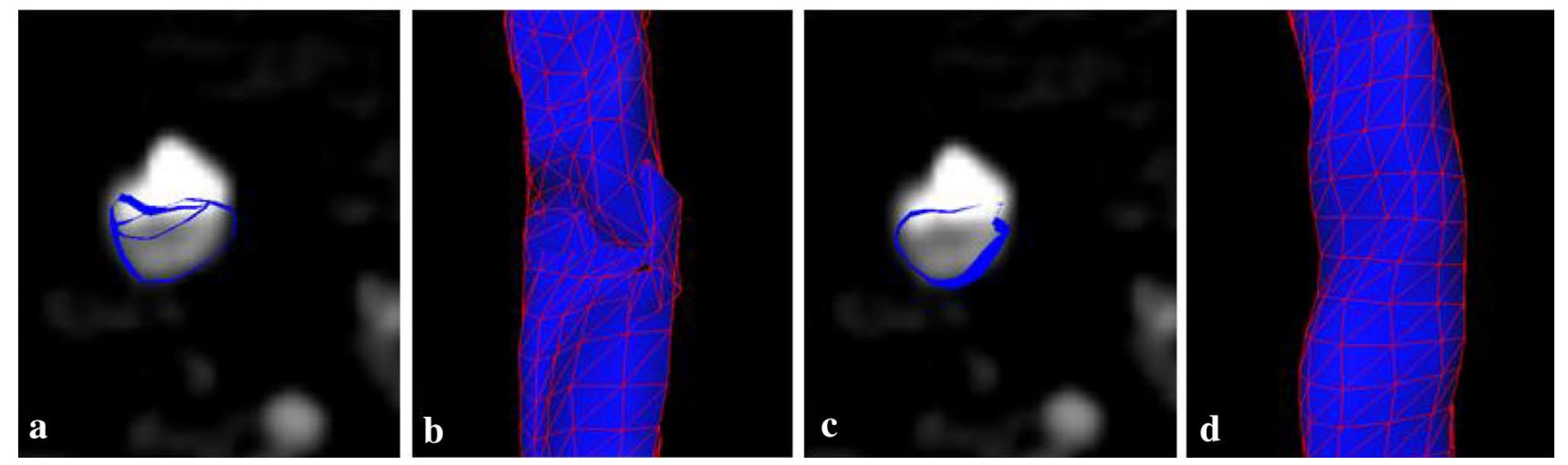

Fig. 5 a Self-intersection in 2D cross-section contour. b 3D surface with self-intersection. $\mathbf{c} 2 \mathrm{D}$ cross section contour and $\mathbf{d}$ 3D surface with no self-intersection when using a non-self-intersection force 
A total of 38 patients underwent pre-operative CTA scanning for TAVR. The baseline characteristics of these patients are listed in Table 1. Two patients were excluded for the following reasons: one patient did not have a whole-body CTA dataset, whereas in the other patient the contrast in the aorto-femoral vessel trajectory was very low.

The datasets were acquired in two hospitals: Leiden University Medical Center (LUMC) in Leiden, the Netherlands, and Fuwai Hospital in Beijing, China. The CTA datasets from LUMC were collected on a 320-row CT scanner (Aquilion ONE, Toshiba Medical System, Japan) by using a helical scan protocol. A bi-phasic injection protocol with intravenous contrast was used: $70 \mathrm{ml}$ contrast $(5 \mathrm{ml} / \mathrm{s})$ and $50 \mathrm{ml}$ saline $(5 \mathrm{ml} / \mathrm{s})$ [19]. The datasets from Fuwai Hospital were acquired on a dual source CT scanner (SOMATOM Definition FLASH, Siemens, Germany) by using a helical scan protocol. A single-phasic injection protocol was used: $350 \mathrm{mgI} / \mathrm{ml}(3-4 \mathrm{ml} / \mathrm{s})$. The axial image size of the wholebody CTA image was $512 \times 512$. In each patient, there were approximately 1000 image slices in the $\mathrm{z}$ axis.

\section{Centerline evaluation}

As was described in [11], in all the 36 patients (100\%) the centerlines were extracted successfully from the common femoral arteries to the sino-tubular junction, inside the lumen of the vessel. The average root mean square error between the automatic

Table 1 Baseline characteristics

\begin{tabular}{ll}
\hline & Total (38) \\
\hline Age (years) & $77 \pm 13$ \\
Gender (\% male) & $21(55)$ \\
Diabetes & $7(18)$ \\
Hypertension $^{\mathrm{a}}$ & $23(61)$ \\
Hypercholesterolemia $^{\mathrm{b}}$ & $21(55)$ \\
Family history of CAD $^{\mathrm{c}}$ & $8(21)$ \\
Smoking & $10(26)$ \\
Obesity $_{\text {Previous PCI }}$ & $4(11)$ \\
Previous CABG & $12(32)$ \\
Previous MI & $10(26)$ \\
\hline
\end{tabular}

Data are represented as mean $\pm \mathrm{SD}$, median (interquartile range) or as number and percentages of patients

$C A B G$ coronary artery bypass graft, $C A D$ coronary artery disease, $M I$ myocardial infarction, $P C I$ percutaneous coronary intervention

a Defined as systolic blood pressure $\geq 140 \mathrm{mmHg}$ and/or diastolic blood pressure $\geq 90 \mathrm{mmHg}$ or the use of antihypertensive medication

b Defined as serum total cholesterol $\geq 230 \mathrm{mg} / \mathrm{dl}$ or serum triglycerides $\geq 200 \mathrm{mg} / \mathrm{dl}$ or treatment with lipid lowering medication

${ }^{c}$ Defined as the presence of coronary artery disease in first-degree family members at age $<55$ years in men and age $<65$ years in women and manual corrected centerlines was $2.55 \pm 0.70 \mathrm{~mm}$ and the average mean error was $1.63 \pm 0.40 \mathrm{~mm}$.

\section{Contour evaluation}

Table 2 shows the Dice similarity results comparisons between the automatic and the observer corrected segmentations for the different parts of the segmented trajectory.

The average Dice similarity indexes between the automatic method and the first observer were $0.977 \pm 0.030$, $0.980 \pm 0.019,0.982 \pm 0.016$ for the left ilio-femoral artery, the right ilio-femoral artery and the aorta, respectively; the average Dice similarity indexes between the automatic method and the second observer were $0.950 \pm 0.040$, $0.954 \pm 0.031$ and $0.965 \pm 0.019$, for the left ilio-femoral artery, the right ilio-femoral artery and the aorta, respectively. The inter-observer variability resulted in a Dice similarity index of $0.954 \pm 0.038,0.952 \pm 0.031$ and $0.969 \pm 0.018$ for the left ilio-femoral artery, the right ilio-femoral artery and the aorta, respectively (Table 2).

To find if there is any correlation between the contour detection and the quality of the datasets, the mean and standard deviation of the $\mathrm{HU}$ value within the descending aorta of each patient were measured and plotted together with the Dice similarity index of the aorta between the automatic system and observer 1 (Fig. 6).

\section{Clinical evaluation}

The most important clinical parameter for the vascular access route is the minimal luminal diameter (MLD). In this study we separated the vascular access route into three segments: the two ilio-femoral arteries and the aorta. The crosssectional diameter was calculated at every point along each centerline segment to build a diameter curve.

For the ilio-femoral access, the diameter and area measurements were taken along the centerline bilaterally with the minimum luminal measurement in both left and right side, including common iliac artery (CIA), external iliac artery (EIA), and common femoral artery (CFA) [4, 8, 20, 21]. For the aorta, the diameter measurements were taken along the centerline from the abdominal aorta until the sino-tubular

Table 2 Performance of automatic segmentation comparing to each of the observers and the observers to each other

\begin{tabular}{llll}
\hline $\begin{array}{l}\text { Dice similarity index } \\
(\text { mean } \pm \text { SD) }\end{array}$ & $\begin{array}{l}\text { Automatic vs } \\
\text { observer 1 }\end{array}$ & $\begin{array}{l}\text { Automatic vs } \\
\text { observer 2 }\end{array}$ & $\begin{array}{l}\text { Observer 1 vs } \\
\text { observer 2 }\end{array}$ \\
\hline $\begin{array}{l}\text { Left ilio-femoral } \\
\text { artery }\end{array}$ & $0.977 \pm 0.030$ & $0.950 \pm 0.040$ & $0.954 \pm 0.038$ \\
$\begin{array}{l}\text { Right ilio-femoral } \\
\text { artery }\end{array}$ & $0.980 \pm 0.019$ & $0.954 \pm 0.031$ & $0.952 \pm 0.031$ \\
Aorta & $0.982 \pm 0.016$ & $0.965 \pm 0.019$ & $0.969 \pm 0.018$ \\
\hline
\end{tabular}


junction, including abdominal aorta, descending aorta and ascending aorta [7].

The mean value, SD and $95 \%$ confidence interval of the parameters are shown in Table 3. The correlation and BlandAltman bias are shown in Table 4. The correlations between automatic group and observer groups were $0.81-0.94$, with $\mathrm{p}$ values smaller than 0.001 . With the commonly used significance level value 0.05 [22], the correlations can be called statistically significant.

The Bland-Altman plots of the minimal ilio-femoral luminal lumen diameter and area are shown in Fig. 7. The mean and SD of the difference between the MLD of the automatic segmentation and the observer 1 segmentation were 0.32 and $0.49 \mathrm{~mm}$, respectively, and for the minimal luminal area (MLA) 2.53 and $5.23 \mathrm{~mm}^{2}$. The mean and SD of the difference between the MLD of automatic segmentation and the observer 2 segmentation were 0.51 and $0.71 \mathrm{~mm}$, respectively, and for the MLA 6.19 and $7.58 \mathrm{~mm}^{2}$. The mean and SD of the difference between the MLD of the observer 1 segmentation and the observer 2 segmentation were 0.18 and $0.71 \mathrm{~mm}$, respectively, and for the MLA 3.66 and $6.89 \mathrm{~mm}^{2}$.
The Bland-Altman plots of the aorta MLD are shown in Fig. 8. The mean and SD of the difference between the MLD of the automatic segmentation and the observer 1 segmentation were 1.03 and $1.41 \mathrm{~mm}$. The mean and SD of the difference between the MLD of the automatic segmentation and the observer 2 segmentation were 1.35 and $1.00 \mathrm{~mm}$. The mean and SD of the difference between the MLD of the observer 1 and the observer 2 segmentation were 0.32 and $1.28 \mathrm{~mm}$.

\section{Discussion}

Over the past few years, the development of TAVR pre-operative planning applications has been driven by the increasing need for proper access route selections and prosthesis size selection during TAVR, and prediction of post-TAVR vascular complications. CT has received a lot of interest because of its $3 \mathrm{D}$ imaging specifications as compared to 2D angiography's limited information [20]. With MPR images, the arterial lumen can be measured accurately in each cross-section, appreciating the elliptical nature of the artery [4, 23, 24]. However, such a manual detection procedure will require too much time and

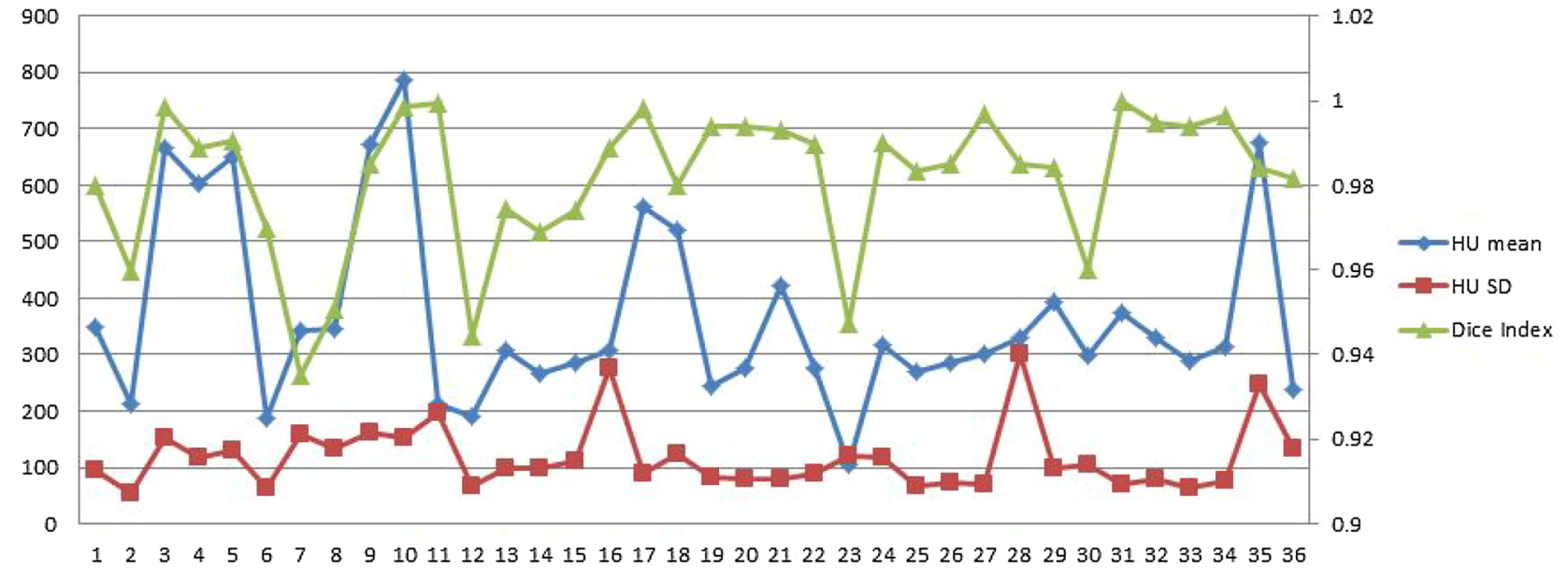

Fig. 6 The image qualities and the contour detection evaluation of the datasets: the blue line is the mean of HU value of the descending aorta, the red is the standard deviation (left vertical axis) and the green is the Dice index (right vertical axis)

Table 3 Results of minimal luminal diameter and area measurements from automatic, the observer 1 and the observer 2 measurement

\begin{tabular}{|c|c|c|c|c|c|c|c|c|c|}
\hline & \multicolumn{3}{|c|}{ Automatic measurement } & \multicolumn{3}{|c|}{ Observer 1 measurement } & \multicolumn{3}{|c|}{ Observer 2 measurement } \\
\hline & Mean & SD & $95 \% \mathrm{CI}$ & Mean & SD & $95 \% \mathrm{CI}$ & Mean & SD & $95 \% \mathrm{CI}$ \\
\hline \multicolumn{10}{|l|}{ Ilio-femoral } \\
\hline Minimal luminal diameter (mm) & 6.03 & 1.48 & $5.53-6.53$ & 5.70 & 1.43 & $5.22-6.19$ & 5.52 & 1.32 & $5.07-5.97$ \\
\hline Minimal luminal area $\left(\mathrm{mm}^{2}\right)$ & 38.43 & 12.95 & $34.04-42.81$ & 35.90 & 12.28 & $31.74-40.05$ & 32.24 & 11.14 & $28.47-36.01$ \\
\hline \multicolumn{10}{|l|}{ Aorta } \\
\hline Minimal luminal diameter ( $\mathrm{mm}$ ) & 13.43 & 2.54 & $12.57-14.29$ & 12.40 & 2.93 & $11.41-13.39$ & 12.08 & 2.40 & $11.27-12.89$ \\
\hline
\end{tabular}


Table 4 Results of minimal luminal diameter and area measurements; comparing the automatic to observer 1 and observer 2, and the observers to each other

\begin{tabular}{llll}
\hline & $\begin{array}{l}\text { Observer 1 vs } \\
\text { automatic }\end{array}$ & $\begin{array}{l}\text { Observer 2 vs } \\
\text { automatic }\end{array}$ & $\begin{array}{l}\text { Observer 1 vs } \\
\text { observer 2 }\end{array}$ \\
\hline $\begin{array}{l}\text { Ilio-femoral } \\
\text { Diameter }\end{array}$ & & \\
$\begin{array}{l}\text { Correlation } \\
\text { Bias }(95 \% \mathrm{CI})\end{array}$ & 0.94 & 0.88 & 0.87 \\
$\mathrm{p}$ value & $-0.32(0.64$ to -1.28$)$ & $-0.51(0.89$ to -1.90$)$ & $0.18(1.57$ to -1.20$)$ \\
Area & $<0.001$ & $<0.001$ & $<0.001$ \\
Correlation & 0.92 & & 0.83 \\
Bias $(95 \% \mathrm{CI})$ & $-2.5(7.7$ to -12.8$)$ & $-6.2(8.7$ to -21.1$)$ & $3.7(17.2$ to -9.9$)$ \\
p value & $<0.001$ & $<0.001$ & $<0.001$ \\
Aorta & & & \\
Diameter & & & 0.91 \\
Correlation & 0.88 & 0.92 & $0.3(2.8$ to -2.2$)$ \\
Bias $(95 \% \mathrm{CI})$ & $-1.0(1.7$ to -3.8$)$ & $-1.3(0.6$ to -3.3$)$ & $<0.001$ \\
p value & $<0.001$ & $<0.001$ & \\
\hline
\end{tabular}

will introduce variabilities. An automatic procedure will be able to reduce the effort and support both inexperienced and experienced observers.

In this paper, a 3D method was introduced for the automatic segmentation of the vessel trajectory for TAVR preoperative planning in whole-body CTA images. To our knowledge, this is the first solution which can automatically segment the whole vessel trajectory from femoral artery, iliac artery, abdominal aorta, descending aorta up to the ascending aorta in a whole-body CTA image data set published in articles.

The whole procedure only requires about $90 \mathrm{~s}$ on a computer with Core i7 3770 and 8 GB RAM with four CPU threads, and can be further optimized. In our procedure, the quantification of the entire aorta-femoral trajectory is implemented, including the ilio-femoral arteries, the thoracic and abdominal aorta. In previous studies on aortic aneurysms, aortic measurement have also been implemented. In the work by Müller-Eschner et al. it took 2.5-5.7 min with purely manually measurement on axial slices, and 4.6-9.3 min on MPR images on the thoracic aorta; with a semi-automatic centerline extraction method, the analysis time was $3.5-9.3 \mathrm{~min}$ [25]. In the study by Kaufmann et al., the mean time to only detect the maximal diameter of the abdominal aorta aneurysm manually was $104.7 \pm 24.9 \mathrm{~s}$ with double-oblique images and $175.2 \pm 100.9 \mathrm{~s}$ to segment semi-automatically the abdominal aorta aneurysm to achieve maximal diameter [26].

In our fully automatic segmentation procedure, a deformable subdivision surface model fitting was used. The deformable subdivision surface model is a new 3D model which processes the entire $3 \mathrm{D}$ data set instead of detecting the 2D transversal contour separately in each 2D image slice or detecting the 2D longitudinal contour on a stretched MPR image. The control points on the subdivision surface are always searching in 3D space for the target boundary of artery. Another method which is similar to our method is the $3 \mathrm{D}$ deformable cylindrical non-uniform rational B-spline (NURBS) model fitting [27]. However, the advantages of deformable subdivision surface model fitting are that it is able to deal with objects with complex topology such as artery bifurcations and it is flexible to deal with complex shapes in the ilio-femoral luminal areas. However, when the model is too flexible, there might be self-intersection of surface. In this study, therefore, a non-self-intersection force was added to overcome this problem. In the future, we believe that the deformable subdivision surface model can also be used to segment other complex anatomical structures, such as the aortic root.

The ability to detect the MLD and MLA of the ilio-femoral arteries in each patient is another important feature of thisapplication. For the pre-operative planning of TF-TAVR, the minimal ilio-femoral artery diameter decides the external sheath size. Post-operatively, vascular access site issues in TF-TAVR procedure are the most common companion disease [28]. It is the main complication in more than $15 \%$ of the patients undergoing TF-TAVR in [4]. Sheath-to-iliofemoral artery ratio (SIFAR), defined as "sheath outer diameter divided by access-side vascular diameter" is known to be predictive of major vascular complications which have high correlation with higher mortality. Whether the TFTAVR is acceptable will depend on the value of SIFAR [20]. In [4], the sheath to artery ratio was calculated by diameter and area, and the area's result seems more reliable. In this study, both diameter and area of the ilio-femoral arteries were calculated, making the patient selection procedure in TAVR pre-operative planning reliable.

Quantitative evaluations were performed in two stages. The first stage was a comparison of the automatic contours 

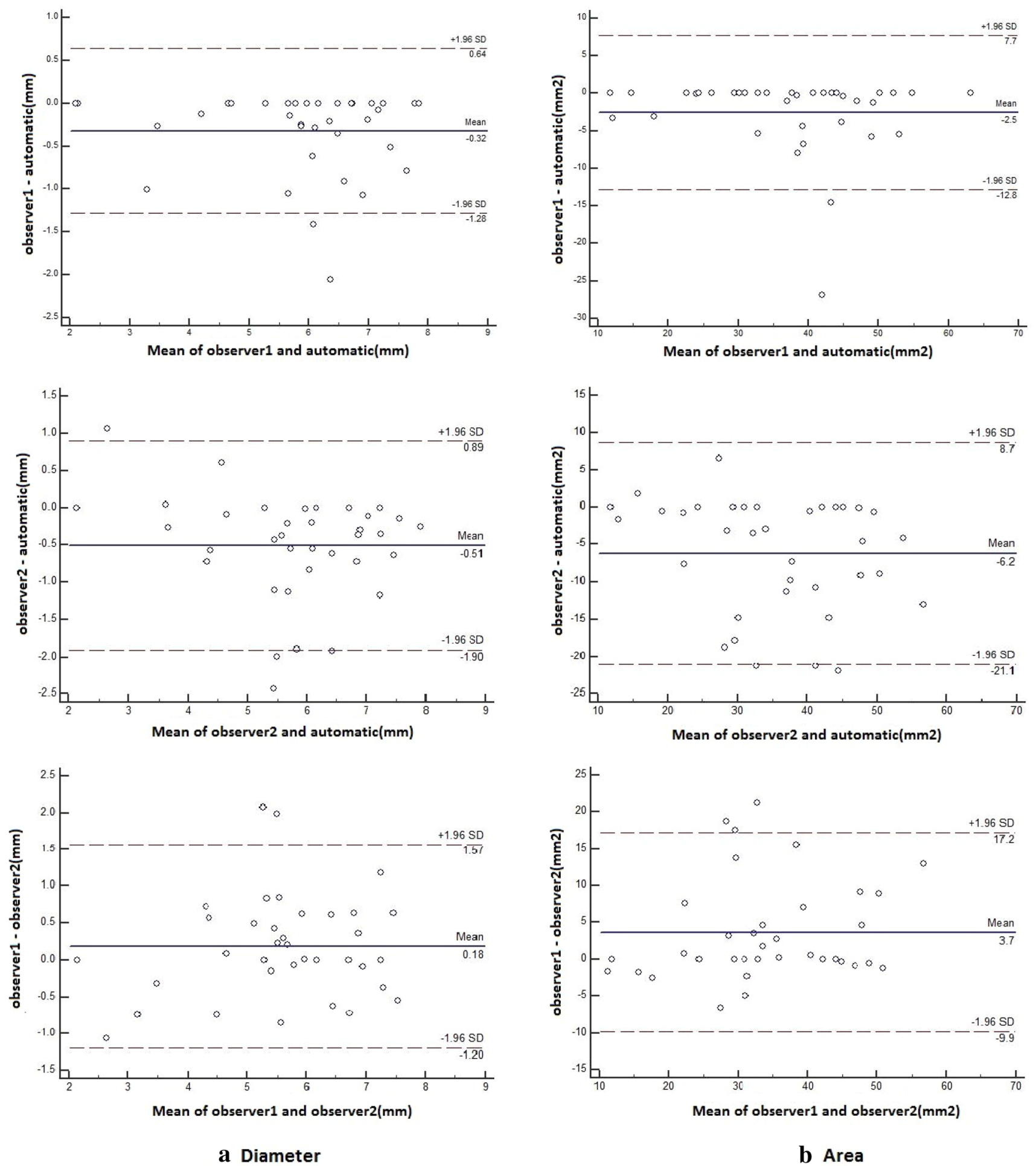

Fig. 7 Bland-Altman plot of minimal ilio-femoral luminal lumen comparing automatic measurement, the observer 1 and the observer 2

with the manually corrected contours. The Dice similarity index in our study was found to be at least 0.95 .

In Fig. 6, the measurement of the Hounsfield units of the contrast in the descending aorta was shown. This provides the indication on the quality of the datasets, which are the amount and the standard deviation of contrast in the aorta. It is apparent that if there is less contrast in the aorta and the contrast is inhomogeneous, the extraction of the aorta will be more difficult. But however, in our procedure, the automatic segmentation results of different datasets were always 

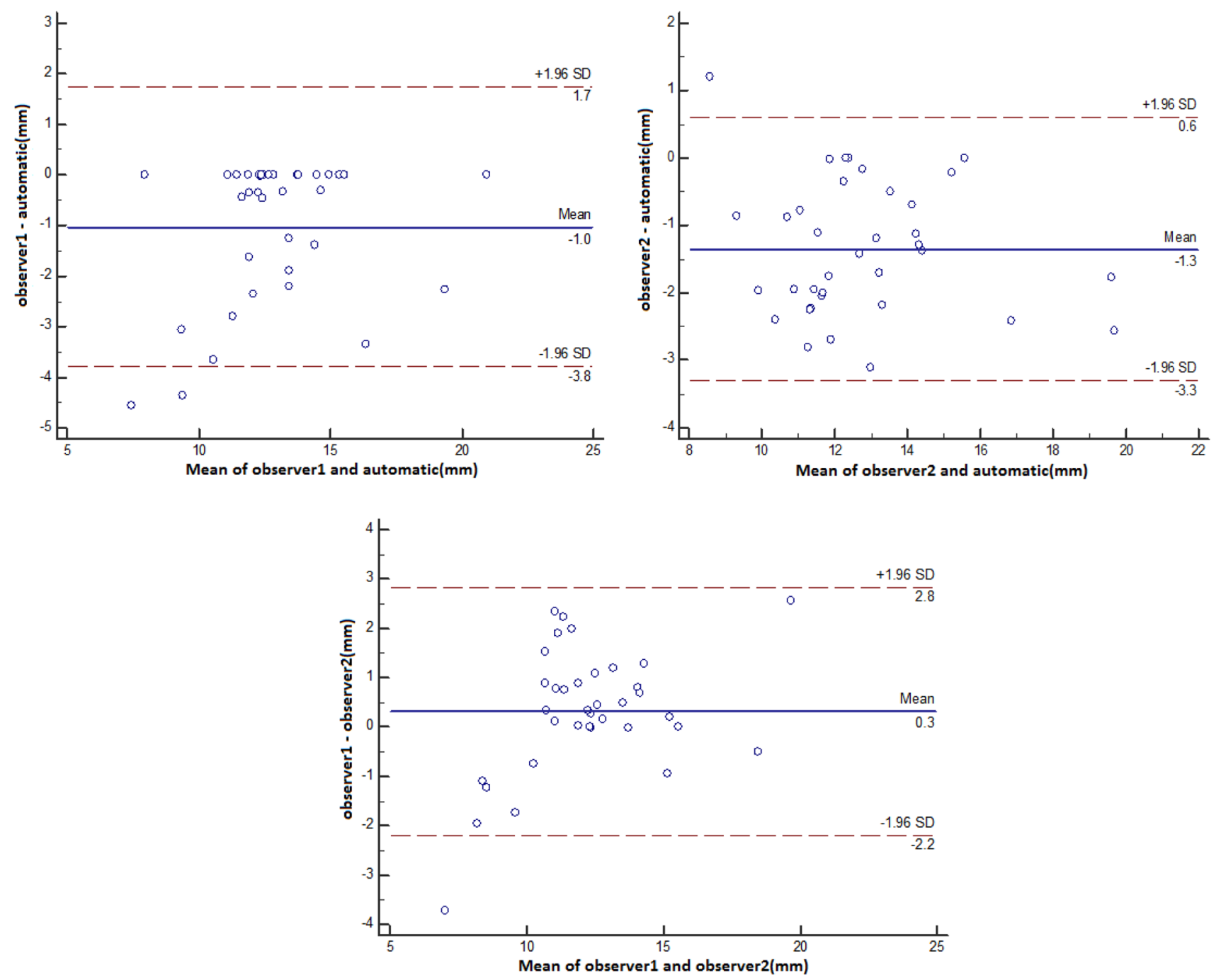

Fig. 8 Bland-Altman plots comparing minimal aorta luminal diameter measurements between automatic, observer 1 and observer 2

good according to Dice similarity index. This proves the robustness of our method.

The second stage was the comparison of clinical parameters from automatic and manually-corrected segmentation. The correlation between the automatic method and the first observer was much higher than the inter-observer variability, and the correlation between the automatic method and the second observer was similar to the inter-observer variability. The result indicates that the automatic method is trustful compared to the manual corrected method. The automatic method overestimated the lumen area and diameter in the ilio-femoral arteries comparing to the results from both observers slightly by around one pixel. The variabilities between the observers of clinical parameters are much higher than the variabilities between automatic measurement and observer 1; the variabilities between the observers of clinical parameters are similar to the variabilities between automatic measurement and observer 2. This means that our technique is more reproducible than between the observers.
In research [29], CTA-based semi-automatic segmentation software was used to measure MLD for TF-TAVR. The result was evaluated by manual results on projection angiography (XA). The difference in MLD between the software and the ground truth was higher than $1.2 \mathrm{~mm}$ in the ilio-femoral artery segments. In our study, the automatic measurement overestimated the MLA only by $0.323 \mathrm{~mm}$ compared to observer 1 , and by $0.51 \mathrm{~mm}$ compared to observer 2, similar to the size of one pixel in the images we used.

In three cases the automatic procedure showed segmentation issue, this can be explained as follows. During the subdivision surface model fitting step, the strongest edge in the intensity image was searched for within certain distance range. This search range was the same for both tiny vessel (such as femoral artery) and larger vessel such as the aorta. Making the search ranges variable for the different vessel sizes should improve the framework and prevent these issues in the future. 


\section{Conclusions}

In conclusion, this automatic TAVR pre-operative application has demonstrated to be able to accurately segment the whole vascular access and measure minimal lumen of the vascular access for TAVR planning in CTA data set.

Acknowledgments Xinpei Gao appreciates the financial support from the China Scholarship Council (No. 201206090162). Shengxian $\mathrm{Tu}$ would like to acknowledge the support by The Program for Professor of Special Appointment (Eastern Scholar) at Shanghai Institutions of Higher Learning and by The Shanghai Pujiang Program (No. 15PJ1404200).

\section{Complaince with ethical standards}

Conflict of interest Pieter Kitslaar is employed by Medis medical imaging systems b.v. and has a research appointment at the Leiden University Medical Center (LUMC). Johan H. C. Reiber is the CEO of Medis medical imaging systems b.v., and has a part-time appointment at LUMC as Prof of Medical Imaging.

Open Access This article is distributed under the terms of the Creative Commons Attribution 4.0 International License (http://creativecommons.org/licenses/by/4.0/), which permits unrestricted use, distribution, and reproduction in any medium, provided you give appropriate credit to the original author(s) and the source, provide a link to the Creative Commons license, and indicate if changes were made.

\section{References}

1. Nkomo VT, Gardin JM, Skelton TN et al (2006) Burden of valvular heart diseases: a population-based study. Lancet 368:10051011. doi:10.1016/S0140-6736(06)69208-8

2. Schoenhagen $P$ et al (2011) Computed tomography in the evaluation for transcatheter aortic valve implantation (TAVI). Cardiovasc Diagn Ther 1(1):44. doi:10.3978/j.issn.2223-3652.2011.08.01

3. Zajarias A, Cribier AG (2009) Outcomes and safety of percutaneous aortic valve replacement. J Am Coll Cardiol 53:1829-1836. doi:10.1016/j.jacc.2008.11.059

4. Krishnaswamy A, Parashar A, Agarwal S, Schoenhagen P et al (2014) Predicting vascular complications during transfemoral transcatheter aortic valve replacement using computed tomography: a novel area-based index. Catheter Cardiovasc Interv 84(5):844-851. doi: $10.1002 / \mathrm{ccd} .25488$

5. Agarwal S, Tuzcu EM, Krishnaswamy A et al (2015) Transcatheter aortic valve replacement: current perspectives and future implications. Heart 101(3):169-177.doi:10.1136/heartjnl-2014-306254

6. Schoenhagen $\mathrm{P}$ et al (2011) Computed tomography evaluation for transcatheter aortic valve implantation (TAVI): imaging of the aortic root and iliac arteries. J Cardiovasc Comput Tomogr 5(5):293-300. doi:10.1016/j.jcct.2011.04.007

7. Achenbach S, Delgado V, Hausleiter J, Schoenhagen P, Min JK, Leipsic JA (2012) SCCT expert consensus document on computed tomography imaging before transcatheter aortic valve implantation (TAVI)/transcatheter aortic valve replacement (TAVR). J Cardiovasc Comput Tomogr 6(6):366-380. doi:10.1016/j. ject.2012.11.002

8. Leipsic J, Gurvitch R, LaBounty TM et al (2011) Multidetector computed tomography in trans-catheter aortic valve implantation.
J Am Coll Cardiol Imaging 4(4):416-429. doi:10.1016/j. jemg.2011.01.014

9. Lesage D, Angelini ED, Bloch I, Funka-Lea G (2009) A review of $3 \mathrm{D}$ vessel lumen segmentation techniques: models, features and extraction schemes. Med Image Anal 13(6):819-845. doi:10.1016/j.media.2009.07.011

10. Kitslaar PH, van't Klooster R, Staring M, Lelieveldt BPF, van der Geest RJ.(2015) Segmentation of branching vascular structures using adaptive subdivision surface fitting. In: Proceedings of the SPIE 9413, Medical Imaging 2015: Image Process, 94133Z.

11. Gao X, Tu S, de Graaf MA, Xu L, Kitslaar P, Scholte AJ, Xu B, Reiber JHC (2014) Automatic extraction of arterial centerline from whole-body computed tomography angiographic datasets. In: Computing in cardiology conference (CinC), pp 697-700

12. Mao SS, Ahmadi N, Shah B, Beckmann D, Chen A, Ngo L, Flores FR, Budoff MJ et al (2008) Normal thoracic aorta diameter on cardiac computed tomography in healthy asymptomatic adults: impact of age and gender. Acad Radiol 15:827-834. doi:10.1016/j.acra.2008.02.001

13. Metz C, Schaap M, Weustink A, Mollet N, van Walsum T, Niessen W (2009) Coronary centerline extraction from CT coronary angiography images using a minimum cost path approach. Med Phys 36:5568-5579. doi:10.1118/1.3254077

14. Gülsün MA, Tek H (2008) Robust vessel tree modeling. Med Image Comput Comput Assist Interv 11:602-611. doi:10.1007/978-3-540-85988-8_72

15. Shahzad R et al (2013) Automatic segmentation, detection and quantification of coronary artery stenoses on CTA. Int J Cardiovasc Imaging 29(8):1847-1859. doi:10.1007/s10554-013-0271-1

16. Steigner ML, Mitsouras D, Whitmore AG, Otero HJ, Wang C, Buckley O, Levit NA, Hussain AZ, Cai T, Mather RT (2010) Iodinated contrast opacification gradients in normal coronary arteries imaged with prospectively ECG-gated single heart beat 320-detector row computed tomography. Circ Cardiovasc Imaging 3(2):179-186. doi:10.1161/CIRCIMAGING.109.854307

17. Loop C (1987) Smooth subdivision surfaces based on triangles. Dissertation, Department of Mathematics, University of Utah

18. Park J-Y, McInerney T, Terzopoulos D, Kim M-H (2001) A non-self-intersecting adaptive deformable surface for complex boundary extraction from volumetric images. Comput Graph 25:421-440. doi:10.1016/S0097-8493(01)00066-8

19. de Graaf FR, Schuijf JD, van Velzen JE et al (2010) Diagnostic accuracy of 320-row multidetector computed tomography coronary angiography to noninvasively assess in-stent restenosis. Invest Radiol 45:331-340. doi:10.1097/RLI.0b013e3181dfa312

20. Okuyama K, Jilaihawi H, Kashif M et al (2015) Transfemoral access assessment for transcatheter aortic valve replacement evidence-based application of computed tomography over invasive angiography. Circ Cardiovasc Imaging 8(1):e001995. doi:10.1161/CIRCIMAGING.114.001995

21. Kurra V et al (2009) Prevalence of significant peripheral artery disease in patients evaluated for percutaneous aortic valve insertion: preprocedural assessment with multidetector computed tomography. J Thorac Cardiovasc Surg 137(5):1258-1264. doi:10.1016/j.jtcvs.2008.12.013

22. Salkind NJ (2006) Encyclopedia of measurement and statistics. Sage, Thousand Oaks

23. Delgado V, Ewe SH, Ng ACT et al (2010) Multimodality imaging in transcatheter aortic valve implantation: key steps to assess procedural feasibility. EuroIntervention 6(5):643-652. doi:10.4244/ EIJV6I5A107

24. Goenka AH et al (2014) Multidimensional MDCT angiography in the context of transcatheter aortic valve implantation. Am J Roentgenol 203(4):749-758. doi:10.2214/AJR.13.12159 
25. Müller-Eschner M, Rengier F, Partovi S et al (2013) Accuracy and variability of semiautomatic centerline analysis versus manual aortic measurement techniques for TEVAR. Eur J Vasc Endovasc Surg 45(3):241-247

26. Kauffmann C, Tang A, Dugas A et al (2011) Clinical validation of a software for quantitative follow-up of abdominal aortic aneurysm maximal diameter and growth by CT angiography. Eur J Radiol 77(3):502-508

27. van't Klooster R, de Koning PJ, Dehnavi RA, Tamsma JT, de Roos A, Reiber JH, van der Geest RJ (2012) Automatic lumen and outer wall segmentation of the carotid artery using deformable three-dimensional models in MR angiography and vessel wall images. J Magn Reson Imaging 35:156-165. doi:10.1002/ jmri.22809
28. Twggweiler S et al (2013) Management of vascular access in transcatheter aortic valve replacement: part 1-basic anatomy, imaging, sheaths, wires, and access routes. J Am Coll Cardiol Cardiovasc Interv 6(7):643-653. doi:10.1016/j.jcin.2013.04.003

29. Wiegerinck EMA et al (2014) Imaging for approach selection of TAVI: assessment of the aorto-iliac tract diameter by computed tomography-angiography versus projection angiography. Int J Cardiovasc Imaging 30(2):399-405. doi:10.1007/ s10554-013-0343-2 